\title{
Microorganisms for analysis
}

\author{
Gérald Thouand
}

Published online: 15 March 2011

(C) Springer-Verlag 2011

The very first observations of microorganisms by Antonie Van Leeuwenhoek and Louis Joblot in the 17th century paved the way to what we now know as modern microbiology. It has been estimated that $50 \%$ of the earth's biomass is microbial (parasites, diatoms, microalgae, fungi, bacteria, and viruses) and of these, viruses (living parasitic organisms that thrive off the cells they infect) have reached an astronomical number $\left(10^{31}\right)$. Humankind and animals are equally dependent on the activity of microorganisms within their environments, because microorganisms are fundamental to all geochemical cycles.

Different observers have perceived microorganisms in different ways. Medical doctors have invariably seen microorganisms as harmful, which is logical when we take into account the history of medicine, whereas biotechnologists have tried to understand and use them in a range of traditional procedures such as bread making and in promising new techniques such as molecule biosynthesis for therapeutic purposes. It is the latter context, i.e. emphasis on the positive side of microorganisms and their function when it comes to analysis (in its broadest sense) within the health sector, the food industry, and the environment sector, that has inspired this special issue.

Microorganisms (mainly bacteria) have been used unequivocally in the field of health in order to assess the

Published in the special issue Microorganisms for Analysis with Guest Editor Gérald Thouand.

G. Thouand $(\bowtie)$

Chargé de Mission Innovation à la DRRT, Auditeur IHEST, UMR

CNRS 6144 GEPEA, Institut Universitaire de Technologie

(Université de Nantes),

Dept GB, 18, Bd. G. Defferre,

85035 La Roche sur Yon, France

e-mail: gerald.thouand@univ-nantes.fr impact of chemotherapeutic agents, as initially discovered by Paul Ehrlich from 1904 onwards. Antibiograms were the first bioassays developed between 1940 and 1945 to determine the activity of newly discovered antibiotics in relation to bacteria test samples which would now be referred to as "sensitive bioelements".

After having achieved incredible scientific and medical advances, researchers during the 1960s became concerned about the effects of chemical substances on the environment and how they could alter key geochemical cycles. The field of ecotoxicology brought about the introduction of a significant number of tests using various typical microbial bioelements to assess the impact of chemical pollutants on their surroundings and their potential impact on microbial ecosystems. We have to acknowledge the work, begun in 1968 by Kempner and Hanson, then continued by Bulich, Isenberg, and Ulitzur in the 1980s, which established the status of a simple bioassay based on the reduced bioluminescence of Vibrio fischeri (now Aliivibrio fischeri), a marine bacterium, when placed in contact with a toxic substance. This test, sold under the name Microtox, is internationally renowned and has without doubt contributed to progress in modern day microbial bioassays.

Besides the effect of toxic substances on the environment, studies published in the 1960s demonstrated the ability of microorganisms to biodegrade organic matter; this led to the publication of articles after 1963 on examination of the measurement of biodegradation. The objective was to assess the biodegradation of chemical substances under the action of microorganisms in a closed reactor (before these substances became available commercially) and to estimate their environmental persistence. This issue continues to be of utmost importance, certainly since the implementation of the European Chemicals Regulation, REACH 1907/2006 (Registration, Evaluation and Authorisation of Chemicals). 
Today's use of microorganisms for analysis is inextricably linked to sensor technology. After the advent of the oxygen sensor in the 1960s, in 1977 Isao Karube from Japan followed the example of the work of Clark and Lyons in 1962 on an enzyme biosensor and successfully immobilised bacteria in an oxygen sensor for the purpose of measuring the biological oxygen demand. This has foreshadowed each successive generation of microbial biosensors. At first sight the idea seems as simple as it is revolutionary-transforming the biological signal (in this case the oxygen consumed by bacteria during consumption of carbon-based organic matter) into an electronic signal by way of an oxygen sensor. At the time, this signal was much easier to measure than determining the quantity of the carbon-based organic matter itself.

1982 was a turning point for microbial biosensors. Two teams, one led by Baldwin the other by Engebrecht, simultaneously published work on cloning bioluminescence genes from Vibrio harveyi and Aliivibrio fischeri into Escherichia coli bacteria, which then became bioluminescent. Since the 1990s, this scientific accomplishment has enabled the development of light-emitting bacteria which react in the presence of some targeted materials (metals, and organic and organometallic chemical substances). The benefit derives from the fact that a specific concentration of a bioavailable pollutant can be measured (that which is in contact with the microorganism), unlike a chemical analysis which measures the total concentration. Chemical and biological analyses are therefore complementary.

What legacy has the first microbial biosensor left us after 30 years? This special issue is devoted to the dynamic use of microorganisms for analysis in science, but it also identifies the negative aspects inherent in this type of procedure (difficulties regarding conservation and immobilisation, and the need for a multi-disciplinary approach to include biologists, physicians, engineers, etc.). Scientific reviews have concentrated on the part played by microbial biosensors in measuring ecotoxicity, in detecting pathogens via bacteriophages, in measuring pollutants in water, but also in using bacterial spores and microalgae, and in highlighting a greater need for microorganisms to be integrated into microsystems. In parallel with these reviews, important and original articles have covered the analytical possibilities of microorganisms for different environmental applications, demonstrating the strong link that exists between biological science and engineering science.

I would like to thank all the authors for their excellent contributions and also the $\mathrm{ABC}$ editorial team for their valuable support throughout the preparation of this special issue.

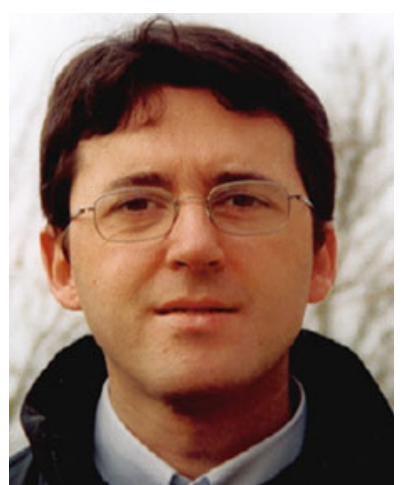

Gérald Thouand

received his $\mathrm{PhD}$ degree in Microbiology from the University of Nancy, France, in 1993. He is a full Professor in Microbiology at the University of Nantes, head of the CBAC Bacterial Sensors for Control and Analysis laboratory (UMR CNRS 6144 GEPEA), representative for research innovation at the French Ministry of Research (DRRT Pays de La Loire), and auditor with the Institute of Higher Studies for Science and Technology (IHEST, Paris).

His research focuses on the environmental monitoring of biodegradation and biotechnology using microbial biosystems. He is primarily involved in the development of biosensors for the detection of chemical pollutants and pathogenic bacteria. A member of the French Society of Microbiology (SFM, France) and the Society for Applied Microbiology (SFAM, UK), he is also associate editor of Frontiers in Microbiology (Frontiers in Microbial Ecotoxicology and Bioremediation). 